\title{
Detecting 22q11.2 deletion in Chinese children with conotruncal heart defects and single nucleotide polymorphisms in the haploid TBX1 locus
}

Yue-Juan $\mathrm{Xu}^{1}$, Jian Wang ${ }^{2}$, Rang Xu ${ }^{3}$, Peng-Jun Zhao ${ }^{1}$, Xi-Ke Wang ${ }^{1}$, Heng-Juan Sun ${ }^{4}$, Li-Ming Bao ${ }^{4,5}$, Jie Shen ${ }^{6}$, Qi-Hua Fu' ${ }^{2}$, Fen $\mathrm{Li}^{1}$ and Kun Sun ${ }^{1,7^{*}}$

\begin{abstract}
Background: Conotruncal heart defects (CTDs) are present in $75-85 \%$ of patients suffering from the $22 q 11.2$ deletion syndrome. To date, no consistent phenotype has been consistently correlated with the $22 \mathrm{q} 11.2$ deletions. Genetic studies have implicated TBX1 as a critical gene in the pathogenesis of the syndrome. The aim of study was to determine the incidence of the 22q11.2 deletion in Chinese patients with CTDs and the possible mechanism for pathogenesis of CTDs.

Methods: We enrolled 212 patients with CTDs and 139 unrelated healthy controls. Both karyotypic analysis and multiplex ligation-dependent probe amplification were performed for all CTDs patients. Fluorescence in situ hybridization was performed for the patients with genetic deletions and their relatives. The TBX1 gene was sequenced for all patients and healthy controls. The $\chi^{2}$ and Fisher's exact test were used in the statistical analysis.

Results: Thirteen of the 212 patients with CTDs (6.13\%) were found to have the 22q11.2 deletion syndrome. Of the 13 cases, 11 presented with a hemizygous interstitial microdeletion from CLTCL1 to LZTR1; one presented with a regional deletion from CLTCL1 to DRCR8; and one presented with a regional deletion from CDC45L to LZTR1. There were eight sequence variants in the haploid TBX1 genes of the del22q11 CTDs patients. The frequency of one single nucleotide polymorphism (SNP) in the del22q11 patients was different from that of the non-del patients ( $P$ $<0.05)$, and the frequencies of two other SNPs were different between the non-del CTDs patients and controls $(P$ $<0.05)$.
\end{abstract}

Conclusions: CTDs, especially pulmonary atresia with ventricular septal defect and tetralogy of Fallot, are the most common disorders associated with the 22q11.2 deletion syndrome. Those patients with both CTDs and 22q11.2 deletion generally have a typical or atypical deletion region within the TBX1 gene. Our results indicate that TBX1 genetic variants may be associated with CTDs.

\section{Background}

Congenital heart defects (CHDs) comprise a group of structural abnormalities with a combined incidence rate of approximately $1 \%$ of all live births. Of the cases of CHDs, $15-20 \%$ are conotruncal heart defects (CTDs) [1]. CTDs share the morphological architecture of the

\footnotetext{
* Correspondence: sunkun@xinhuamed.edu.cn

'Department of Pediatric Cardiology, Shanghai Children's Medical Center Affiliated to Shanghai Jiaotong University School of Medicine, Shanghai 200127, China

Full list of author information is available at the end of the article
}

presence of ventricular outflow tract defects. They include tetralogy of Fallot (TOF), pulmonary atresia with ventricular septal defect (PA/VSD), double outlet of right ventricular (DORV), transposition of the great arteries (TGA), persistent truncus arteriosus (PTA) and interrupted aortic arch (IAA).

Although CTDs are generally considered complex, multifactorial disorders, a number of familial cases suggesting Mendelian inheritance have been described. The DiGeorge/Velo-cardio-facial syndrome (DGS/VCFS) is a

\section{Biomed Central}


genetic condition related to the 22q11.2 deletion, and $75-85 \%$ of the deletion patients present with a CTD. Reports originating from Western countries associate $12.8-17.8 \%$ of their CTD cases to the $22 \mathrm{q} 11.2$ deletion [2-5]. Most of the 22q11.2 deletions are sporadic in origin; however, approximately $10 \%$ of the deletions are inherited [6]. In total, $84-90 \%$ of the patients have a common region of deletion that is approximately $3 \mathrm{Mb}$ deletion, which contains about 40 genes; while $7-14 \%$ present with a smaller, less common proximal $\sim 1.5 \mathrm{Mb}$ nested deletion, containing about 30 genes [7]. The deletions are reported to occur as a result of a non-allelic homologous recombination between the low-copy repeats (LCRs) in the $22 \mathrm{q} 11.2$ region $[8,9]$. A minority of patients have smaller overlapping and non-overlapping 22q11.2 deletions [10].

The $T B X 1$ gene maps to the $1.5 \mathrm{Mb}$ typically deleted region associated with DGS/VCFS and encodes a transcription factor of the T-box family known to play an important role in the regulation of developmental processes [11]. A TBX1 haploinsufficiency is thought to be responsible for many of the phenotypic traits of the DGS/VCFS. Mutations of the TBX1 gene have been detected in some patients featuring DGS/VCFS who are otherwise devoid of the 22q11.2 deletion [12-14].

Our previous screening for 22q11.2 deletions in 24 Chinese patients with CTDs by fluorescence in situ hybridization (FISH) showed a lower incidence rate $(4.2 \%)$ of del22q11 than that usually reported (12.8$17.8 \%)[2-5,15]$. In order to better understand the incidence of del22q11 in Chinese patients with CTDs, we carried out this current study in a new population of 212 patients.

\section{Methods}

\section{Subjects}

All assessments were undertaken with the approval of the Medical Ethics Committee of the Shanghai Children's Medical Center (SCMC). Patients were recruited prospectively from June 2008 to December 2009 at the SCMC. A total of 212 patients with CTDs were enrolled, of whom 92 were female and 120 male. The median age was 2.69 years old, and all of the patients were of the Han ethnicity. They included 74 TOF, 51 DORV, 35 PA/VSD, 28 TGA, 4 IAA and 3 PTA patients as well as 17 other cases of conotruncal malformations (Figure 1). Diagnoses were confirmed by transthoracic echocardiography and cardiac catheterization. When available, the surgical operative notes were reviewed. Extracardiac anomalies were also evaluated. Informed written parental consents were obtained for all of the patients participating in the study before blood samples were drawn at the time of catheterization.

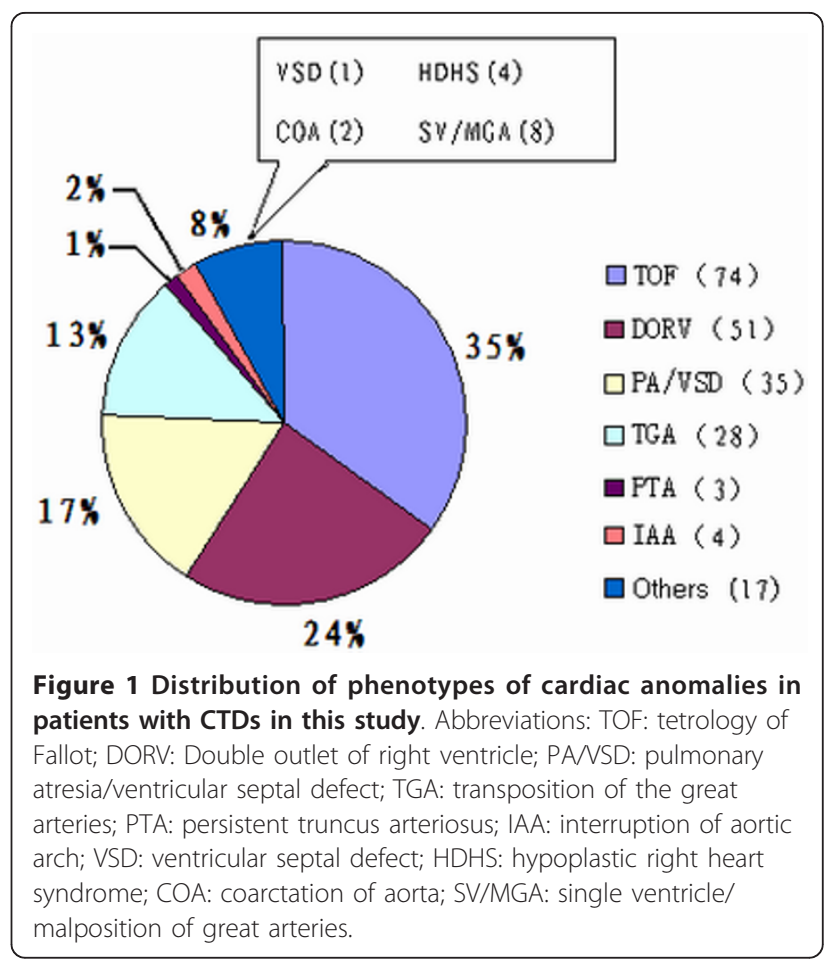

Normal controls included 139 unrelated healthy Chinese children (57 female, 82 male; all ethnically Han) for detecting genetic variations at the TBX1 locus. The median age was 7.43 years old. The cardiac morphology of these controls was confirmed to be normal by transthoracic echocardiography at the SCMC. After obtaining informed written parental consent, a peripheral venous blood sample was collected into an anticoagulation tube with sodium citrate. Genomic DNA was extracted from peripheral lymphocytes using a QIAamp DNA Blood Mini Kit in keeping with the manufacturer's instructions (Qiagen, Duesseldorf, Germany).

\section{Cell culture and karyotyping}

The karyotypes were examined using GTG-banding at a 400 band level. The lymphocytes obtained from the peripheral blood of the patients were cultured at $37^{\circ} \mathrm{C}$ with $5 \% \mathrm{CO}_{2}$. The slide preparation for karyotyping was performed using the cultured peripheral blood cells. The G-banding was performed on these slides using a modification of Seabright's technique [16]. In each case, at least 20 banded metaphases with good chromosome separation were analyzed by experienced geneticists.

\section{Multiplex ligation-dependent probe amplification (MLPA)}

Genomic DNA was extracted from peripheral lymphocytes using a QIAamp DNA Blood Mini Kit according to the manufacturer's instructions (Qiagen). For detection of the 22q11.2 deletion, we used a commercial 
SALSA P250-A1 MLPA-DiGeorge syndrome test kit (MRC-Holland, Amsterdam, The Netherlands). The P250-A1 MLPA probemix contains 48 probes for the frequent 22q11 deletion regions and the infrequent deletion regions. These probes are capable of distinguishing the most common types of deletion, 30 of which are related to the chromosomal region of 22q11. Five probes cover the Cat Eye Syndrome (CES) region, and another 14 fall on the most common DiGiorge deletion region. The 30 probes hybridize to a $7.3 \mathrm{Mb}$ genomic region on chromosome 22q11. The most commonly deleted region starts at probe CLTCL1, downstream of LCR-A, and ends at probe LZTR1, upstream of LCR-D. The genomic distance between probes CLTCL1 and LZTR1 is approximately 2.2-2.5 $\mathrm{Mb}$ (Figure 2). The remaining 18 probes are located on 22q13,10p15, 8p23, 4q34-qter, 9q34.3 and 17p13.3.

The MLPA reactions were performed following the manufacturer's instructions on all CTDs patients. After denaturation, hybridization, ligation and amplification, the polymerase chain reaction (PCR) products were sequenced by capillary electrophoresis using an $\mathrm{ABI}$ 3130 Genetic Analyzer (Applied Biosystems, Foster City, CA, USA). The raw data from electrophoresis were imported into the GeneMapper 4.0 software program (Applied Biosystems) and then analyzed using the Coffalyser MLPA DAT software package (MRC-Holland). After normalization, the data were compared to a

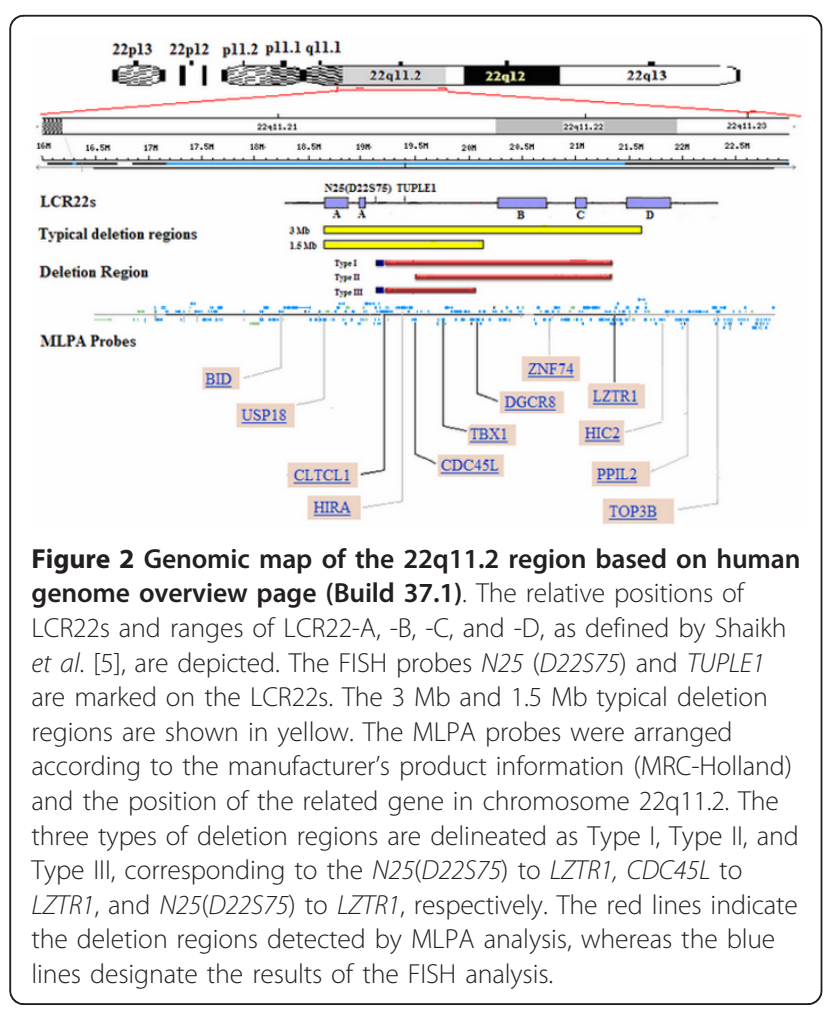

synthetic control sample, which represents the median of all normal samples in each experiment. A threshold of signal intensity change of $<0.75$ was used to identify potential deletions, and a threshold of $>1.33$ was used to identify potential duplications. All samples with potential deletions or duplications were re-analyzed by MLPA.

\section{FISH}

The 22q11.2 deletions detected in patients by MLPA were confirmed by FISH analysis. Commercially available locus specific probe kits N25 and TUPLE1 were purchased from Vysis (Downers Grove, IL, USA). The N25 kit contains the spectrum orange N25 (D22S75) probe for 22q11.2 and the spectrum green ARSA gene control probe for 22q13.3. The TUPLE1 kit contains the spectrum orange TUPLE1 (HIRA) probe at 22q11.2 and the spectrum green TelVysion 22q probe at 22q13.3.

The FISH probes N25 (D22S75) and TUPLE1 (HIRA) both lie within the DiGeorge chromosomal region (DGCR). The probe TUPLE1 is located between CLTCL1 and D22S941 and overlaps with the P250-A1 MLPA probe HIRA in the chromosome. The N25 (D22S75) probe lies between the P250-A1 MLPA probe DGCR2 and CLTCL1 (Figure 2).

Dual-color FISH was performed on metaphase spreads of peripheral blood lymphocytes on pre-cleaned glass slides. Co-denaturation of probes and slides was performed at $73^{\circ}$ $\mathrm{C}$ for $5 \mathrm{~min}$, followed by overnight hybridization at $42^{\circ} \mathrm{C}$. After hybridization and washing, chromosomes and nuclei were counterstained with DAPI (Vysis, Downers Grove, IL, USA). A coverslip was then applied to each slide. Images were captured using a CCD camera (ProgRes, Jena, Germany) through an Olympus-BX51 fluorescence microscope (Olympus, Japan) with the VideoTest Fish 2.0 software package (GP Medical Technologies Ltd, Beijing, China). Generally, 10 to 20 metaphases were examined, and 30 to 50 interphase nuclei were scored for the number of signals present for each probe.

\section{Gene PCR and sequencing}

The three different splice variants of the TBX1 coding regions-including exon-intron boundaries and the promoter site-were amplified in the 212 CTD patients and 139 unrelated healthy controls. Primers were designed following the corresponding genomic regions available from the GenBank database (NG_009229.10) using Primer3 software (http://frodo.wi.mit.edu/primer3/). All PCR products were sequenced on an ABI 3130 sequencer (Applied Biosystems). The sequence traces were aligned with the reference sequence using the GenBank BLAST program.

\section{Statistical analysis}

The Hardy-Weinberg equilibrium (HWE) and allele frequencies were assessed at each single nucleotide 
polymorphism (SNP) locus using the $\chi^{2}$ and Fisher's exact test. HWE_P value $>0.05$ was considered as a statistically insignificant deviation from the equilibrium. The 2-sided statistical tests were considered significant with a level of $P \leq 0.05$. All statistical analyses in our study were carried out with SPSS 11.5 (SPSS Inc. Chicago, IL, USA).

\section{Results}

\section{Cell culture and karyotyping}

No obvious structural and numerical abnormalities were found on the metaphase spreads of the 212 patients.

\section{Multiplex ligation-dependent probe amplification}

MLPA screening identified a loss of DNA dosage in chromosome $22 \mathrm{q} 11$ in 13 subjects $(6.13 \%)$ out of the 212 CTD patients screened (Table 1), an incidence lower than that reported (12.8-17.8\%) by authors in the West [2-5]. Of these 13 subjects, 11 were found to have an identical deletion region about 2.2-2.5 $\mathrm{Mb}$ in size, spanning from probe CLTCL1 to LZTR1 (LCR22-A to LCR22-D) (Figures 2 and 3). Patient NO.10D differed in that he showed a deletion region about 1.8-2.0 $\mathrm{Mb}$ in size, from CDC45L to LZTR1 (atypical LCR22-A to LCR22-D) (Figures 2 and 3). In patient NO.13, we found yet a smaller deletion region about $0.9-1.3 \mathrm{Mb}$ in size, extending from CLTCL1 to DGCR8 (LCR22-A to LCR22-B) (Figures 2 and 3). Other probes assaying for deletions in chromosome 4p, 8p, 9p, 10p and 17p did not reveal obvious changes in the number of copies.

\section{FISH}

Of the 13 patients with deletions identified by MLPA, 12 were confirmed by using two FISH probes. FISH examination of their family members indicated that the

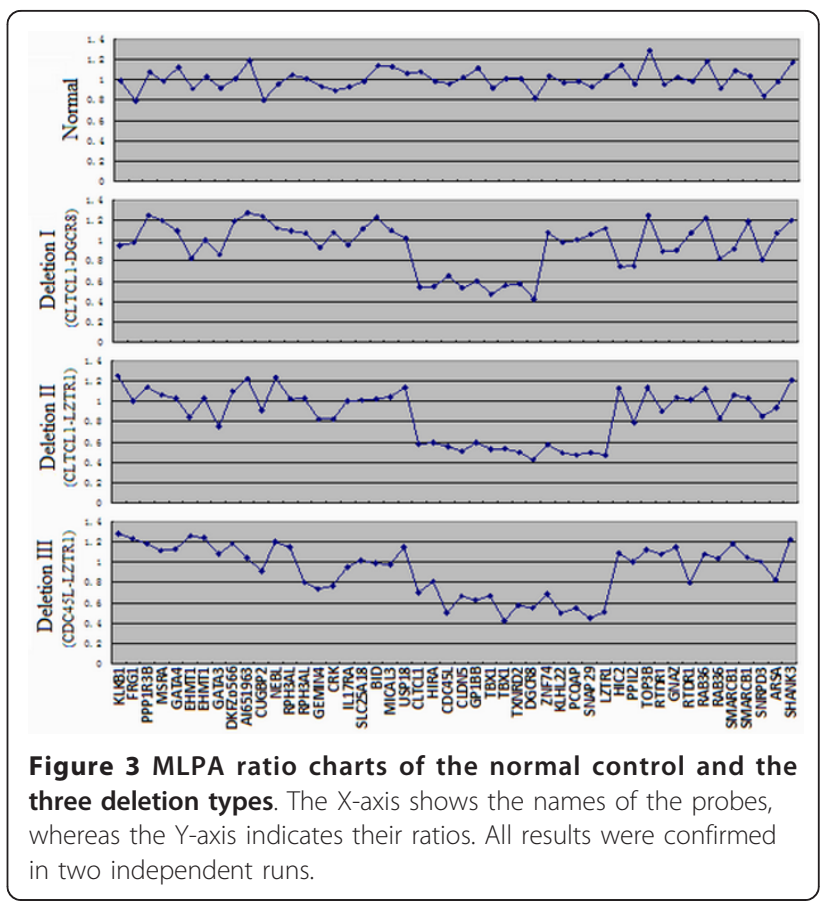

deletions arose de novo. In patient NO.10D, with a deletion region from $C D C 45 L$ to $L Z T R 1$, the signals of both probes $N 25$ (D22S75) and TUPLE1 were detected in the FISH slides (data not shown).

From the combined results of MLPA and FISH, two of the deletion regions could be redefined from $N 25$ (D22S75) to LZTR1 and from N25 (D22S75) to DGCR8. The proximal breakpoint of the deletion region from $C D C 45 L$ to LZTR1 may be located on or neighboring CDC45L, which is at locus $0.03 \mathrm{Mb}$ to HIRA (TUPLE1) (Figure 2).

Table 1 Detailed information of the 13 CTD patients with 22q11.2 deletion

\begin{tabular}{|c|c|c|c|c|c|c|c|}
\hline \multirow[t]{2}{*}{ NO. } & \multirow{2}{*}{$\begin{array}{l}\text { Age* }^{*} \\
(\mathrm{~m})\end{array}$} & \multirow{2}{*}{$\begin{array}{c}\text { Cardiac Defects } \\
\text { Primary Diagnosis }\end{array}$} & \multirow[t]{2}{*}{ Karyotype } & \multirow{2}{*}{$\begin{array}{c}\text { Size of } \\
\text { Deletion (MLPA) }\end{array}$} & \multicolumn{2}{|c|}{ FISH } & \multirow{2}{*}{$\begin{array}{c}\text { TBX1 } \\
\text { Sequence }\end{array}$} \\
\hline & & & & & $\mathrm{N} 25$ & TUPLE1 & \\
\hline T36 & 21 & TOF & $46, X Y$ & CLTCL1-LZTR1 & Del & Del & $\mathrm{H}$ \\
\hline $1 \mathrm{E}$ & 13 & TOF & $46, X X$ & CLTCL1-LZTR1 & Del & Del & $\mathrm{H}$ \\
\hline $2 \mathrm{~F}$ & 19 & TOF & $46, X Y$ & CLTCL1-LZTR1 & Del & Del & $\mathrm{H}$ \\
\hline 33 & 24 & TOF & $46, X X$ & CLTCL1-LZTR1 & Del & Del & $\mathrm{H}$ \\
\hline 18 & 8 & PANSD & $46, X Y$ & CLTCL1-LZTR1 & Del & Del & $\mathrm{H}$ \\
\hline $1 \mathrm{C}$ & 4 & PANSD & $46, X X$ & CLTCL1-LZTR1 & Del & Del & $\mathrm{H}$ \\
\hline 32 & 23 & PANSD & $46, X Y$ & CLTCL1-LZTR1 & Del & Del & $\mathrm{H}$ \\
\hline 11 & 15 & PANSD & $46, X Y$ & CLTCL1-LZTR1 & Del & Del & $\mathrm{H}$ \\
\hline $2 \mathrm{D}$ & 23 & PANSD & $46, X X$ & CLTCL1-LZTR1 & Del & Del & $\mathrm{H}$ \\
\hline 5 & 9 & PANSD & $46, X Y$ & CLTCL1-LZTR1 & Del & Del & $\mathrm{H}$ \\
\hline $10 \mathrm{D}$ & 120 & PANSD & $46, X Y$ & CDC45L-LZTR1 & $\mathrm{N}$ & $N$ & $\mathrm{H}$ \\
\hline D2 & 21 & DORV & $46, X Y$ & CLTCL1-LZTR1 & Del & Del & $\mathrm{H}$ \\
\hline 13 & 8 & PTA(A1) & $46, X X$ & CLTCL1-DGCR8 & Del & Del & $\mathrm{H}$ \\
\hline
\end{tabular}


Table 2 Clinical manifestations of the 13 CTD patients with 22q11.2 deletion

\begin{tabular}{cccccc}
\hline NO. & Age* & Size of & \multicolumn{2}{c}{ Cardiac defects } & Extracaridac anomalies \\
\cline { 4 - 5 } & $(\mathbf{m})$ & Deletion & P.D. & S.D. & Nil \\
\hline T36 & 21 & D22S75-LZTR1 & TOF & PFO/RAA & Asymmetric cry/smile face \\
$1 E$ & 13 & D22S75-LZTR1 & TOF & PFO/APCA & Nil \\
$2 F$ & 19 & D22S75-LZTR1 & TOF & PFO/RAA/APCA & Nil \\
33 & 24 & D22S75-LZTR1 & TOF & ASD(II) & Hypoplastic thymus \\
18 & 8 & D22S75-LZTR1 & PANSD & PDA/ASD(II) & Cleft palate \\
$1 C$ & 4 & D22S75-LZTR1 & PANSD & PFO/APCA & Classic dysmorphological facial features; hypoplastic thymus \\
32 & 23 & D22S75-LZTR1 & PANSD & PFO/PDA/APCA & Nil \\
11 & 15 & D22S75-LZTR1 & PANSD & PDA & Classic dysmorphological facial features \\
$2 D$ & 23 & D22S75-LZTR1 & PANSD & PDA/ASD/MAPCA & inguinal hernia \\
5 & 9 & D22S75-LZTR1 & PANSD & ASD(II)/MAPCA & Nil \\
$10 D$ & 120 & CDC45L-LZTR1 & PANSD & PDA/ASD/APCA & Nil \\
D2 & 21 & D22S75-LZTR1 & DORV & VSD/PS & Nil \\
13 & 8 & D22S75-DGCR8 & PTA(A1) & VSD &
\end{tabular}

Abbreviations: * age in months at the point of examination; $m=$ months P.D. = primary diagnosis; S.D. = secondary diagnosis; ASD (II) = secondary atrial septal defect; $\mathrm{APCA}=$ aortic-pulmonary collateral arteries; MAPCA = major aortic-pulmonary collateral artery; PDA = patent ductus arteriosus; PFO = Patent Foramen Ovale; RAA = right aortic arch; PS = pulmonary stenosis.

\section{Assessment of clinical manifestation}

Table 2 lists the clinical manifestations (cardiac and extracardiac anomalies) of the 13 CTD patients determined to have the 22q11.2 deletion, including seven patients with PA/VSD, four with TOF, one with PTA and one with DORV; meanwhile, none of the TGA and IAA patients had such deletions. Of the patients with deletions, eight suffered from right aortic arch (RAA) or aortopulmonary collateral arteries (APCAs), and six from extracardiac anomalies.

\section{Gene PCR and sequencing}

In the 22q11.2 deletion group, no heterozygotes of $T B X 1$ were detected, which is in accordance with the partial monosomy for $22 \mathrm{q} 11.2$. No mutations were detected in the haploid TBX1 gene. However, eight different sequence variants were detected in the haploid TBX1 gene of the 13 del22q11 patients (Figure 4). The allele frequencies of SNPs observed in our study were similar to that observed in the Han Chinese of 1000 Genome Project Database (http://browser.1000genomes.

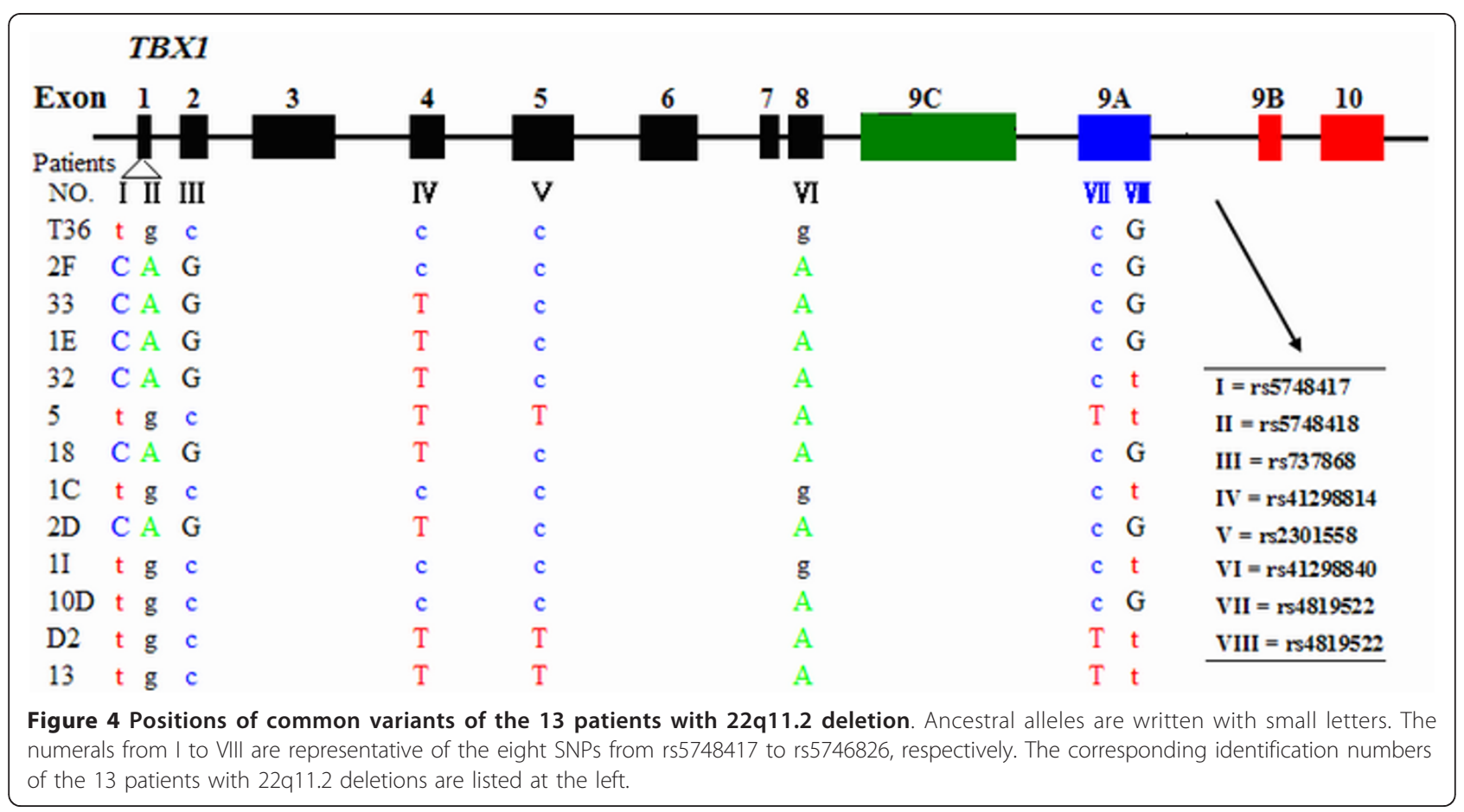


org). All eight SNPs of HWE_P values were $>0.05$ in the non-deletion patients and healthy controls. All of the substitutions were common variants with a minor allele frequency (MAF) of $>0.05$. All variants, except for rs41298840 (c.933A > G, A311A), were detected at similar frequencies between del22q11 and non-del CTD patients (Table 3). The frequencies of polymorphisms
c.933A > G were similar between the non-del22q11 CTD patients and the healthy controls, but they differed from that of the del22q11 CTD patients $(P<0.05)$ (Table 3).

The frequencies of rs5748417 (c.-87+242T $>\mathrm{C}$ ) and rs5748418 (c.-87+256G > A), as shown in Table 4, were similar between the del22q11 CTD patients and

Table 3 Allele frequencies of SNPs in del22q11 patients $(n=13)$, non-del patients $(n=199)$, controls $(n=139)$ and Han Chinese in 1000 Genome Project $(n=197)$

\begin{tabular}{|c|c|c|c|c|c|c|c|c|}
\hline \multirow[t]{2}{*}{ SNP } & \multicolumn{2}{|c|}{${ }^{\$} A F(\%(n))$} & \multicolumn{2}{|c|}{${ }^{\$} A F(\%(n))$} & \multicolumn{2}{|c|}{${ }^{\$} A F(\%(n))$} & \multicolumn{2}{|c|}{${ }^{\$} A F(\%(n))$} \\
\hline & del & non-del & non-del & control & del & control & control & ${ }^{\#}$ 1000GPD \\
\hline \multicolumn{9}{|l|}{ rs5748417 } \\
\hline $\mathrm{T}$ & $53.8(7)$ & $68.6(273)$ & $68.6(273)$ & $59.0(164)$ & $53.8(7)$ & $59.0(164)$ & $59.0(164)$ & $66.0(260)$ \\
\hline C & $46.2(6)$ & $31.4(125)$ & $31.4(125)$ & $41.0(114)$ & $46.2(6)$ & $41.0(114)$ & $41.0(114)$ & $34.0(134)$ \\
\hline$\chi^{2}$ & \multicolumn{2}{|c|}{1.261} & \multicolumn{2}{|c|}{6.600} & \multicolumn{2}{|c|}{0.136} & \multicolumn{2}{|c|}{3.427} \\
\hline${ }^{*} P(\mathrm{df}=1)$ & \multicolumn{2}{|c|}{0.363} & \multicolumn{2}{|c|}{0.011} & \multicolumn{2}{|c|}{0.777} & \multicolumn{2}{|c|}{0.074} \\
\hline \multicolumn{9}{|l|}{ rs5748418 } \\
\hline G & $53.8(7)$ & $72.9(290)$ & $72.9(290)$ & $59.0(164)$ & $53.8(7)$ & $59.0(164)$ & $59.0(164)$ & $66.0(260)$ \\
\hline A & $46.2(6)$ & 27.1(108) & $27.1(108)$ & $41.0(114)$ & $46.2(6)$ & $41.0(114)$ & $41.0(114)$ & $34.0(134)$ \\
\hline$\chi^{2}$ & \multicolumn{2}{|c|}{2.272} & \multicolumn{2}{|c|}{14.28} & \multicolumn{2}{|c|}{0.136} & \multicolumn{2}{|c|}{3.427} \\
\hline${ }^{*} P(\mathrm{df}=1)$ & \multicolumn{2}{|c|}{0.203} & \multicolumn{2}{|c|}{$2.0 \mathrm{E}-4$} & \multicolumn{2}{|c|}{0.777} & \multicolumn{2}{|c|}{0.074} \\
\hline \multicolumn{9}{|l|}{ rs737868 } \\
\hline G & $46.2(6)$ & $31.7(126)$ & $31.7(126)$ & 38.8(108) & $46.2(6)$ & 38.8(108) & 38.8(108) & 39.8(157) \\
\hline C & $53.8(7)$ & $68.3(272)$ & $68.3(272)$ & $61.2(170)$ & $53.8(7)$ & $61.2(170)$ & $61.2(170)$ & $60.2(237)$ \\
\hline$\chi^{2}$ & & & & & & & & \\
\hline${ }^{*} P(\mathrm{df}=1)$ & & & & & & & & \\
\hline rs41298814 & & & & & & & & \\
\hline $\mathrm{T}$ & $61.5(8)$ & $46.2(184)$ & $46.2(184)$ & $51.8(144)$ & $61.5(8)$ & $51.8(144)$ & $51.8(144)$ & $51.5(203)$ \\
\hline C & $38.5(5)$ & $53.8(214)$ & $53.8(214)$ & $48.2(134)$ & $38.5(5)$ & $48.2(134)$ & $48.2(134)$ & 48.5(191) \\
\hline$\chi^{2}$ & & & & & & & & \\
\hline${ }^{*} P(\mathrm{df}=1)$ & & & & & & & & \\
\hline rs2301558 & & & & & & & & \\
\hline $\mathrm{T}$ & 23.1(3) & 14.8(59) & 14.8(59) & $14.4(40)$ & 23.1(3) & $14.4(40)$ & $14.4(40)$ & $12.4(49)$ \\
\hline C & $76.9(10)$ & $85.2(339)$ & 85.2(339) & $85.6(238)$ & $76.9(10)$ & $85.6(238)$ & $85.6(238)$ & $87.6(345)$ \\
\hline$\chi^{2}$ & & & & & & & & \\
\hline${ }^{*} P(\mathrm{df}=1)$ & & & & & & & & \\
\hline rs41298840 & & & & & & & & \\
\hline A & $76.9(10)$ & $44.7(178)$ & $44.7(178)$ & 47.1(131) & $76.9(10)$ & 47.1(131) & 47.1(131) & $52.3(206)$ \\
\hline G & 23.1(3) & $55.3(220)$ & $55.3(220)$ & $52.9(147)$ & 23.1(3) & $52.9(147)$ & $52.9(147)$ & 47.7(188) \\
\hline$\chi^{2}$ & & & & & & & & \\
\hline${ }^{*} P(\mathrm{df}=1)$ & & & & & & & & \\
\hline rs4819522 & & & & & & & & \\
\hline C & $76.9(10)$ & $86.3(344)$ & $86.4(344)$ & $86.3(240)$ & $76.9(10)$ & $86.3(240)$ & $86.3(240)$ & $87.1(343)$ \\
\hline $\mathrm{T}$ & 23.1(3) & $13.6(54)$ & $13.6(54)$ & 13.7(38) & 23.1(3) & 13.7(38) & 13.7(38) & $12.9(51)$ \\
\hline$\chi^{2}$ & & & & & & & & \\
\hline${ }^{*} P(d f=1)$ & & & & & & & & \\
\hline rs5746826 & & & & & & & & \\
\hline G & $53.8(7)$ & $33.9(135)$ & $33.9(135)$ & $38.1(106)$ & $53.8(7)$ & 38.1(106) & $38.1(106)$ & $37.6(148)$ \\
\hline $\mathrm{T}$ & $46.2(6)$ & $66.1(263)$ & $66.1(263)$ & $61.9(172)$ & $46.2(6)$ & $61.9(172)$ & $61.9(172)$ & $62.4(246)$ \\
\hline$\chi^{2}$ & & & & & & & & \\
\hline${ }^{*} P(\mathrm{df}=1)$ & & & & & & & & \\
\hline
\end{tabular}

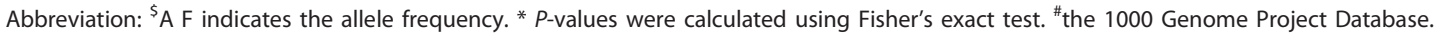


Table 4 Allele frequencies of rs41298838 (c.928G > A, G310S) in the three groups

\begin{tabular}{|c|c|c|c|c|c|c|}
\hline \multirow[t]{2}{*}{ SNP } & \multicolumn{2}{|c|}{ Allele Frequency $(\%(n))$} & \multicolumn{2}{|c|}{ Allele Frequency $(\%(n))$} & \multicolumn{2}{|c|}{ Allele Frequency $(\%(n))$} \\
\hline & del & non-del & non-del & control & del & control \\
\hline G & $100(13)$ & $95.7(382)$ & $95.7(382)$ & $97.5(271)$ & $100(13)$ & $97.5(271)$ \\
\hline A & $0(0)$ & $4.3(17)$ & $4.3(17)$ & $2.5(7)$ & $0(0)$ & $2.5(7)$ \\
\hline$\chi^{2}$ & \multicolumn{2}{|c|}{1.578} & \multicolumn{2}{|c|}{1.455} & \multicolumn{2}{|c|}{0.335} \\
\hline${ }^{*} P(\mathrm{df}=1)$ & \multicolumn{2}{|c|}{1.000} & \multicolumn{2}{|c|}{0.292} & \multicolumn{2}{|c|}{1.000} \\
\hline
\end{tabular}

* P-values were calculated using Fisher's exact test.

controls. However, they were significantly different between the non-del22q11 CTD patients and controls (Table 3). The discrepancy may be due to the limited sample size $(n=13)$ of the patients with CTDs and del22q11. Further analysis of the three genotype frequencies of the two SNPs between non-del22q11 CTD patients and controls showed significant differences between the two groups at both genetic polymorphisms (Table 5).

In addition, the non-synonymous SNP rs41298838 (c.928G > A, G310S) was found to occur at a similar frequency in both the non-del22q11 patients and healthy controls (Table 4). This result is in contrast with a previous finding by Yagi et al. [12], who reported it as a mutation. It is, however, in line with the study by Heike et al., who used SIFT and Polyphen to predict that this nonsynonymous SNP would be tolerated by the protein [17].

\section{Discussion}

Microdeletion in the $22 \mathrm{q} 11$ region causes a variety of disorders, including the DiGeorge (DGS; OMIM 188400) and Velo-cardio-facial (VCFS; OMIM 192430) syndrome. Summarized as a deletion syndrome

Table 5 Genotype frequencies of rs5148417 and rs5148418 in non-del patients $(n=199)$, controls $(n=$ 139 ) and Han Chinese in 1000 Genome Project ( $n=197)$

\begin{tabular}{|c|c|c|c|c|}
\hline \multirow[t]{2}{*}{ SNP } & \multicolumn{2}{|c|}{ Genotype frequency $(\%(n))$} & \multicolumn{2}{|c|}{ Genotype frequency $(\%(n))$} \\
\hline & Non-del & control & control & 1000GPD \\
\hline \multicolumn{5}{|l|}{ rs5748417 } \\
\hline$\Pi$ & 48.7(97) & $35.3(49)$ & $35.3(49)$ & $44.2(87)$ \\
\hline TC & $39.7(79)$ & $47.5(66)$ & $47.5(66)$ & $43.7(86)$ \\
\hline CC & $11.6(23)$ & $17.3(24)$ & $17.3(24)$ & $12.2(24)$ \\
\hline HWE_P & 0.801 & 1.000 & 1.000 & 0.975 \\
\hline$\chi^{2}$ & \multicolumn{2}{|c|}{6.522} & \multicolumn{2}{|c|}{3.337} \\
\hline${ }^{*} P(\mathrm{df}=2)$ & \multicolumn{2}{|c|}{0.038} & \multicolumn{2}{|c|}{0.189} \\
\hline \multicolumn{5}{|l|}{ rs5748418 } \\
\hline GG & $50.3(100)$ & $35.3(49)$ & $35.3(49)$ & $44.2(87)$ \\
\hline GA & $40(80)$ & $47.5(66)$ & $47.5(66)$ & $43.7(86)$ \\
\hline $\mathrm{AA}$ & $9.5(19)$ & $17.3(24)$ & $17.3(24)$ & $12.2(24)$ \\
\hline HWE_P & 1.000 & 1.000 & 1.000 & 0.975 \\
\hline$\chi^{2}$ & \multicolumn{2}{|c|}{9.013} & \multicolumn{2}{|c|}{3.337} \\
\hline${ }^{*} P(\mathrm{df}=2)$ & \multicolumn{2}{|c|}{0.011} & \multicolumn{2}{|c|}{0.189} \\
\hline
\end{tabular}

* P-values were calculated using Fisher's exact test.
(22q11DS), it is the most frequent chromosomal microdeletion syndrome, occurring once in 4,000 live births [18]. Applying FISH with commercially available N25 (D22S75) or TUPLE1 probes located in the proximal commonly deleted region of $22 \mathrm{q} 11.2$ is a proven method for detection of the $22 \mathrm{q} 11$ deletion. Most of the $22 \mathrm{q} 11.2$ deletions can be found using FISH diagnostic probes, although this method has failed to detect deletions that are either proximal or distal to the FISH probes used $[7,19,20]$. Methods employing multiple genetic markers in the $22 q 11$ region are increasingly important for the accurate identification of genomic microdeletions. MLPA analysis with multiple probes has been applied to analyze the 22q11 chromosome in detail [21], and the commercially available high-definition MLPA 22q11 kit has proven to be a sensitive tool for detecting copy number changes on the long arm of chromosome 22. In our study, in one patient (NO.10D) who tested negative for the 22q11.2 deletion using FISH probes N25 (D22S75) and TUPLE1, MLPA analysis actually identified an atypical deletion from CDC45L to CLTCL1.

In general, CTDs are the disorders most commonly associated with the 22q11.2 deletion syndrome [22,23]. Of the study subjects, $20 \%(7 / 35)$ of those with PA/ VSD, 5.4\% (4/74) with TOF, 33\% (1/3) with PTA and $2 \%$ (1/51) with DORV were detected to have the 22q11.2 deletion. None of the patients with TGA had the $22 \mathrm{q} 11.2$ deletion. In the subgroup of patients presenting with the 22q11.2 deletion, 53.8\% (7/13) suffered from PA/VSD, 30.8\% (4/13) from TOF, and 69.2\% from RAA or APCAs (Table 1 and 2). This result is in line with the findings of previous studies, which concluded that a high prevalence of the deletion is noted in patients with TOF, PA/VSD, PTA, as well as RAA and major APCAs [23,24]. Despite its commonness in patients with the $22 \mathrm{q} 11.2$ deletion in previous studies [25-27], none of the patients with the IAA condition examined in this study showed this deletion. This finding may be due to the small number $(n=4)$ of IAA patients investigated or to possible differences in the 22q11DS pathoanatomy between Western and Asian populations. Further investigation of the deletion in a larger sample size of IAA patients is necessary to clarify this issue. 
To date, multiple phenotypic features and associated abnormalities have been observed in patients with the 22q11.2 deletion. However, no consistent correlation between the genotype and phenotype has been shown for this syndrome. Rauch et al. conjectured that conotruncal cardiac defects related to the 22q11.2 deletion are usually associated with the common $3 \mathrm{Mb}$ or 1.5 $\mathrm{Mb}$ proximally nested deletions, both of which involve the TBX1 gene [11]. This study shows that all deletion types are found in the commonly deleted region, implicating the involvement of the TBX1 gene. However, a detailed analysis of the clinical manifestations did not reveal any genotype-phenotype correlations in the subjects of our study (Table 2).

$T B X 1$ is thought to be a major candidate gene that influences the cardiac phenotype or its severity in patients carrying the 22q11.2 deletion [28]. Its dosedependent role in heart development has been demonstrated in mouse models [29]. Therefore, screening of the TBX1 gene on the residual 22q11 homologous chromatid would likely indicate whether the sequence variants or mutations of the remaining TBX1 copy would have an impact on the phenotype, thereby revealing possible correlations between the TBX1 genotype and cardiac phenotype. No mutations were found in this group of patients, but eight sequence variants were found in the haploid TBX1 gene. When comparing the del22q11 CTD patients to the non-del CTDs patients, the frequencies of polymorphism c.933A > G (rs41298840) in the del22q11 patients significantly differed from that in the non-del22q11 CTD patients and healthy controls $(P$ $<0.05$ ). The non-synonymous SNP rs41298838 (c.928G $>$ A, G310S), which is a known mutation [12] previously predicted to be a tolerant polymorphism [16], was found in both the non-del22q11 CTD patients and healthy controls at similar frequencies. Prior studies have found that although the TBX1 $1^{\mathrm{G} 310 \mathrm{~S}}$ mutation does not prevent transactivation, it does prevent the TBX1-SMAD1 interaction [30,31], which may have had an impact on the homeostasis of the cardiac progenitor cells [32]. These differences imply that the loci rs41298838 and rs41298840 may have an impact on the pathogenesis of this syndrome. However, the findings presented may be biased due to the small number of del22q11 patients studied.

The allele and genotype frequencies of SNPs rs5748417 (c.-87+242T > C) and rs5748418 (c.-87+256G $>$ A) were significantly different between the nondel22q11 CTDs patients and controls. The alleles c. -87 $+242 \mathrm{~T}$ and c. $-87+256 \mathrm{G}$ were more frequent in the group of non-del22q11 patients with CTDs than in controls. These differences suggest the association of these SNPs in the pathogenesis of CTDs. However, further studies are needed to clarify the mechanism, especially since both of the SNPs are located within the 5'UTR intron of the $T B X 1$ gene.

\section{Conclusions}

CTDs, especially PA/VSD and TOF, are the most common disorders associated with the 22q11.2 deletion syndrome. MLPA using commercial kits is a rapid, reliable, and cost-effective method for the diagnosis of 22q11DS. Almost all del22q11 CTD patients identified in this study have a deletion region (typical or atypical) in the $T B X 1$ gene. The differences in allele and genotype frequency distributions of rs5748417 and rs5748418 between non-del22q11 CTD patients and controls imply that the genetic variation in the $T B X 1$ gene may be associated with CTDs. Although the $T B X 1$ gene polymorphisms on the residual 22q11 homologous chromatid have not been found to be a major modifier for the pathogenesis of 22q11.2 deletion syndrome in previous studies [33,34], the fact that frequencies of the SNPs rs41298838 and rs41298840 in del22q11.2 CTD patients differed from those in the non-del CTDs patients in this study indicated that polymorphisms of the residual $T B X 1$ gene may have an impact on the pathogenesis of this syndrome. Further studies with larger sample sizes are warranted to determine if this notion holds true.

\section{Acknowledgements and Funding}

We thank Jiang Wenting, Tao jiong, Wang Lirong, Wu YiMing and Yu YongGuo for their excellent technical assistance, Elfie Mason and Sian Tsuei for their critical reading of the manuscript. The project was funded by a grant (81070135/H0204) from the National Natural Science Foundation of China, the Shanghai University Innovation Team (Phase I): Pediatrics, the Key Discipline of Shanghai Municipal Education Commission Major (Phase III): Pediatric Cardiology, and the grant from Shanghai Shen Kang Hospital Development Center (SHDC 12007201).

\section{Author details}

${ }^{1}$ Department of Pediatric Cardiology, Shanghai Children's Medical Center Affiliated to Shanghai Jiaotong University School of Medicine, Shanghai 200127, China. 'Medical Laboratory, Shanghai Children's Medical Center Affiliated to Shanghai Jiaotong University School of Medicine, Shanghai 200127, China. ${ }^{3}$ Medical Laboratory, Scientific Research Center, Xinhua Hospital Affiliated to Shanghai Jiaotong University School of Medicine, Shanghai 200092, China. ${ }^{4}$ Medical Laboratory, Shanghai Children's Hospital Affiliated to Shanghai Jiaotong University School of Medicine, Shanghai 200040, China. ${ }^{5}$ Division of Human Genetics, Cincinnati Children's Hospital Medical Center and University of Cincinnati College of Medicine, Cincinnati, Ohio 45229, USA. 'Department of Pediatric Cardiology, Shanghai Children's Hospital Affiliated to Shanghai Jiaotong University School of Medicine, Shanghai 200040, China. ${ }^{7}$ Department of Pediatric Cardiology, Xinhua Hospital Affiliated to Shanghai Jiaotong University School of Medicine, Shanghai 200092, China.

\section{Authors' contributions}

YJX designed the study, collected the samples, analyzed the data and drafted the manuscript. JW helped to screen the individuals and collect samples, and participated in analyzing the data. RX participated in the design of the study and helped to draft the manuscript. PJZ and XKW collected the samples. HJS and LMB helped to analyze the data. JS, QHF, and FL participated in the design of the study. KS conceived of the study, participated in its design and coordination, analyzed the data and helped draft the manuscript. All authors read and approved the final manuscript. 


\section{Competing interests}

The authors declare that they have no competing interests.

Received: 10 March 2011 Accepted: 21 December 2011

Published: 21 December 2011

\section{References}

1. Conti E, Grifone N, Sarkozy A, Tandoi C, Marino B, Digilio MC, Mingarelli R, Pizzuti A, Dallapiccola B: DiGeorge subtypes of nonsyndronmic conotruncal defects: evidence against a major role of TBX1 gene.. Eur J Hum Genetic 2003, 11(4):349-351.

2. Derbent M, Yilmaz Z, Baltaci V, Saygili A, Varan B, Tokel K: Chromosome 22q11.2 deletion and phenotypic features in 30 patients with conotruncal heart defects. Am J Med Genet 2003, 116A(2):129-135.

3. Devriendt K, Eyskens B, Swillen A, Dumoulin M, Gewillig M, Fryns JP: The incidence of a deletion in chromosome 22q11 in sporadic and familial conotruncal heart disease. Eur J Pediatr 1996, 155(8):721.

4. Goldmuntz E, Clark BJ, Mitchell LE, Jawad AF, Cuneo BF, Reed L, McDonaldMcGinn D, Chien P, Feuer J, Zackai EH, Emanuel BS, Driscoll DA: Frequency of 22q11 deletions in patients with conotruncal defects. J Am Coll Cardiol 1998, 32(2):492-498.

5. Marino B, Digilio MC, Toscano A, Giannotti A, Dallapiccola B: Congenital heart defects in patients with DiGeorge/velocardiofacial syndrome and del22q11. Genet Couns 1999, 10(1):25-33.

6. McDonald-McGinn DM, Tonnesen MK, Laufer-Cahana A, Finucane B, Driscoll DA, Emanuel BS, Zackai EH: Phenotype of the 22q11.2 deletion in individuals identified through an affected relative: cast a wide FISHing net! Genet Med 2001, 3(1):23-29.

7. Rauch A, Zink S, Zweier C, Thiel CT, Koch A, Rauch R, Lascorz J, Hüffmeier U, Weyand M, Singer H, Hofbeck M: Systematic assessment of atypical deletions reveals genotype-phenotype correlation in 22q11.2. Med Genet 2005, 42(11):871-876.

8. Shaikh TH, Kurahashi H, Saitta SC, O'Hare AM, Hu P, Roe BA, Driscoll DA, McDonald-McGinn DM, Zackai EH, Budarf ML, Emanuel BS: Chromosome 22-specific low copy repeats and the 22q11.2 deletion syndrome: genomic organization and deletion endpoint analysis. Hum Mol Genet 2000, 9(4):489-501.

9. Edelmann L, Pandita RK, Spiteri E, Funke B, Goldberg R, Palanisamy N, Chaganti RS, Magenis E, Shprintzen RJ, Morrow BE: A common molecular basis for rearrangement disorders on chromosome 22q11. Hum Mol Genet 1999, 8(7):1157-1167.

10. Stachon AC, Baskin B, Smith AC, Shugar A, Cytrynbaum C, Fishman L, Mendoza-Londono R, Klatt R, Teebi A, Ray PN, Weksberg R: Molecular diagnosis of 22q11.2 deletion and duplication by multiplex ligation dependent probe amplification. Am J Med Genet Part A 2007, 143A(24):2924-2930.

11. Wilson V, Conlon FL: The T-box family. Genome Biology 2002, 3(6):3008.1-3008.7.

12. Yagi H, Furutani Y, Hamada H, Sasaki T, Asakawa S, Minoshima S, Ichida F, Joo K, Kimura M, Imamura S, Kamatani N, Momma K, Takao A, Nakazawa M, Shimizu N, Matsuoka R: Role of TBX1 in human del 22q11.2 syndrome. Lancet 2003, 362(9393):1366-1373.

13. Lindsay EA, Vitelli F, Su H, Morishima M, Huynh T, Pramparo T, Jurecic V, Ogunrinu G, Sutherland HF, Scambler PJ, Bradley A, Baldini A: TBX1 haploinsufficieny in the DiGeorge syndrome region causes aortic arch defects in mice. Nature 2001, 410(6824):97-101.

14. Paylor R, Glaser B, Mupo A, Ataliotis P, Spencer C, Sobotka A, Sparks C, Choi CH, Oghalai J, Curran S, Murphy KC, Monks S, Williams N, O'Donovan MC, Owen MJ, Scambler PJ, Lindsay E: TBX1 haploinsufficiency is linked to behavioral disorders in mice and humans: implications for 22q11 deletion syndrome. Proc Natl Acad Sci 2006, 103(20):7729-7734.

15. Shen $L, X u Y J$, Zhao PJ, Sun K: Frequency of $22 q 11$ deletions in children with isolated conotruncal defects. Chin J Contemp Pediatr 2009, 11(1):25-28.

16. Seabright M: A rapid banding technique for human chromosomes. Lancet 1971, 2:971-972.

17. Heike $C L$, Starr JR, Rieder MJ, Cunningham ML, Edwards KL, Stanaway IB, Crawford DC: Single nucleotide polymorphism discovery in TBX1 in individuals with and without 22q11.2 deletion syndrome. Birth Defects Res A 2010, 88(1):54-63.
18. Schinke M, Izumo S: Deconstructing DiGeorge syndrome. Nat Genet 2001, 27(3):238-240.

19. Amati F, Conti E, Novelli A, Bengala M, Digilio MC, Marino B, Giannotti A, Gabrielli O, Novelli G, Dallapiccola B: Atypical deletions suggest five 22q11.2 critical regions related to the DiGeorge/velo-cardio-facial syndrome. Eur J Hum Genetic 1999, 7(8):903-909.

20. Beaujard MP, Chantot S, Dubois M, Keren B, Carpentier W, Mabboux P Whalen S, Vodovar M, Siffroi JP, Portnoï MF: Atypical deletion of 22q11.2: Detection using the FISH TBX1 probe and molecular characterization with high-density SNP arrays. Eur J Hum Genetic 2009, 52(2):321-327.

21. Yang C, Huang CH, Cheong ML, Hung KL, Lin LH, Yu YS, Chien CC, Huang HC, Chen CW, Huang CJ: Unambiguous molecular detections with multiple genetic approach for the complicated chromosome 22q11 deletion syndrome. BMC Med Genet 2009, 25:10:16.

22. Kessler-Icekson G, Birk E, Weintraub AY, Barhum Y, Kotlyar V, Schlesinger $H$, Rockah R, Vidne BA, Frisch A: Association of tetralogy of Fallot with a distinct region of del22q11.2. Am J Med Genet 2002, 107(4):294-298.

23. Momma K: Cardiovascular anomalies associated with chromosome 22q11.2 Deletion Syndrome. Am J Cardiol 2010, 105(11):1617-1624.

24. Rauch R, Hofbeck M, Zweier C, Koch A, Zink S, Trantmann U, Hoyer J, Kaulitz R, Singer H, Rauch A: Comprehensive genotype - phenotype analysis in 230 patients with tetralogy of Fallot. J Med Genet 2010, 47(5):321-331.

25. Botto LD: A population-based study of the 22q11.2 deletion: phenotype, incidence, and contribution to major birth defects in the population. Pediatr 2003, 112(1 Pt 1):101-107.

26. Khositseth $A$, Tocharoentanaphol $C$, Khowsathit $P$, Ruangdaraganon N: Chromosome 22q11 deletion in Patients with Conotruncal heart defects. Pediatr Cardiol 2005, 26(5):570-573.

27. Boudjemline Y, Fermont L, Le Bidois J, Lyonnet S, Sidi D, Bonnet D: Prevalence of $22 q 11$ deletion in fetuses with conotruncal cardiac defects: a 6-year prospective study. J Pediatr 2001, 138(4):520-524.

28. Baldini A: DiGeorge syndrome: the use of model organisms to dissect complex genetics. Hum Mol Genet 2002, 11(20):2363-2369.

29. Zhang $Z$, Baldini A: In vivo response to high-resolution variation of $T B X 1$ mRNA dosage. Hum Mol Genet 2008, 17(1):150-157.

30. Zweier C, Sticht H, Aydin-Yaylagul I, Campbell CE, Rauch A: Human TBX1 missense mutations cause gain of function resulting in the same phenotype as 22q11.2 deletions. Am J Hum Genet 2007, 80(3):510-517.

31. Stoller JZ, Epstein JA: Identification of a novel nuclear localization of signal in $T B X 1$ that is deleted in DiGeorge syndrome patients harboring the 1223delC mutation. Hum Mol Genet 2005, 14(7):885-892.

32. Fulcoli FG, Huynh T, Scambler PJ, Baldini A: TBX1 regulates the BMPSmad1 pathway in a transcription independent manner. PlOS ONE 2009, 4(6):e6049.

33. Rauch A, Devriendt K, Koch A, Rauch R, Gewillig M, Kraus C, Weyand M, Singer $\mathrm{H}$, Reis $\mathrm{A}$, Hofbeck M: Assemment of association between variants and haplotypes of the remaining TBX1 gene and manifestations of congenital heart defects in 22q11.2 deletion patients. J Med Genet 2004, 41(4):e40.

34. Voelckel MA, Girardot L, Giusiano B, Levy N, Philip N: Allelic variations at the haploid TBX1 locus do not influence the cardiac phenotype in cases of 22q11 microdeletion. Ann Genet 2004, 47(3):235-240.

\section{Pre-publication history}

The pre-publication history for this paper can be accessed here: http://www.biomedcentral.com/1471-2350/12/169/prepub

doi:10.1186/1471-2350-12-169

Cite this article as: Xu et al:: Detecting 22q11.2 deletion in Chinese children with conotruncal heart defects and single nucleotide polymorphisms in the haploid TBX1 locus. BMC Medical Genetics 2011 12:169. 